\title{
Tri-Concomitant Local Feature Learning for Diabetic Retinopathy Classification
}

\author{
Santosh Nagnath Randive, Ranjan Kumar Senapati
}

\begin{abstract}
In this paper, we have proposed a new technique entitled as Transformed Directional Tri Concomitant Triplet Patterns with Artificial Neural Network is proposed for Diabetic Retinopathy Classification. TdtCTp consist of three stages to obtain detail directional information about pixel progression. In first stage, structural rule based approach is proposed to extract directional information in various direction. Further, in second stage, microscopic information and correlation between each sub-structural element are extracted by using concomitant conditions. Finally, minute directional intensity variation information and correlation between the sub-structural elements are extracted by integrating first two stages. After feature extraction, the extracted feature is used as input to the artificial neural network. To the best of our knowledge, this is the first learning based approach for diabetic retinopathy classification. Effectiveness of the proposed method is evaluated in terms of average precision and compared with existing state-of-the-art methods. The experimental analysis shows that the proposed method is achieved significant performance compared to other methods.
\end{abstract}

Keywords: Feature extraction, artificial neural network, Diabetic Retinopathy Classification.

\section{INTRODUCTION}

In the field of biomedical imaging, there is large increment in biomedical data due to the advanced technique like X-ray, magnetic resonance imaging, computed tomography. Manual access, search, indexing and retrieval of this huge data is difficult task. Thus, there is a need to develop well-structured technique to overcome above mentioned limitation. Feature extraction plays vital role in accuracy of any image retrieval system. In diabetic retinopathy (DR), the progressive damage of retina vison occurs subsequently to occur the complete blindness. DR development, genetic constraints, and metabolic control density are the prior constraints to associate with the growth of the blindness as DR distresses the blood vessels in the retina. In primary stage, the supply routes in retina begin to outflow, and little hemorrhages are

Revised Manuscript Received on December 30, 2019.

* Correspondence Author

Santosh Nagnath Randive*, Research Scholar, Department of Electronics \& Communication Engineering, Koneru Lakshmaiah Education Foundation, Green Fields, Vaddeswaram, Guntur, Andhra Pradesh 522502 India,

Ranjan Kumar Senapati, Professor, Department of Electronics \& Communication Engineering, Koneru Lakshmaiah Education Foundation, Green Fields, Vaddeswaram, Guntur, Andhra Pradesh 522502 India,

(C) The Authors. Published by Blue Eyes Intelligence Engineering and Sciences Publication (BEIESP). This is an open access article under the CC BY-NC-ND license (http://creativecommons.org/licenses/by-nc-nd/4.0/) shaped. The vessels that are spilling from residuals of lipoproteins make a haze vision. A consequent issue is the advancement of weak veins which crack and leaks out blood into the eye, and thus, the retina couldn't extend pictures to the brain, which brings about visual impairment and blindness. In existing literature, both local and global feature descriptor are proposed for feature extraction. Local feature descriptor divides the input image into number of local parts followed by spatial and transform domain features extraction. Whereas, global feature descriptor make use of entire image for visual feature

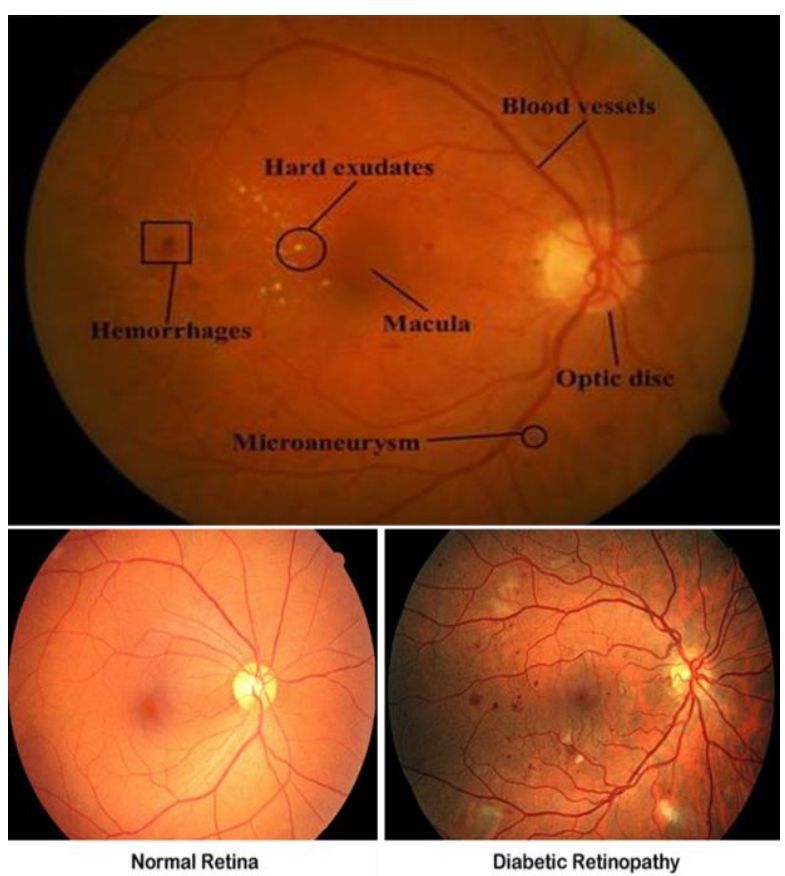

Fig. 1. Basic details of retinal image and structure of Normal and Diabetic Retinopathy images.

extraction. Pictorial illustrations are basically shape; colors including textures are utilized as a fundamental tool for clinical raw databases in the image processing for human discrimination which accomplishes beneficial features for computer based machine intelligence. One of the popular health issue experience by humans is Diabetes. This is also belongs to the category of common disease that affects the health of human beings. From a recent survey by International Diabetes Federation, it is stated that the count of diabetic patients will increase to 592 million by 2035. In this manuscript: Section I illustrates the introduction of Diabetic Retinopathy (DR). Section II describes the related work for DR classification. Section III depicts proposed system framework for DR classification with the help of extracted feature is discussed. 


\section{Tri-Concomitant Local Feature Learning for Diabetic Retinopathy Classification}

Result analysis is discussed in Section IV. Finally, Section V concludes the proposed approach for diabetic retinopathy classification.

\section{RELATED WORK}

Actuality, DR [2] [3] is the most hurtful ophthalmic circumstance enticed by DM in the event that it couldn't be recognized before and fittingly treated [1]. By means of DR [2] [3] is a significant elucidation of vision defeat between individuals suffering from diabetes [4], they must be observed

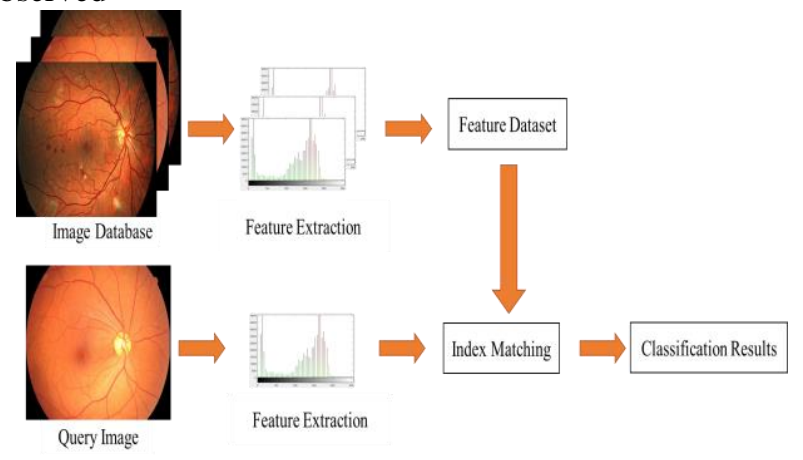

Fig. 2. Block diagram of the generalized classification approach for image.

at ordinary interims in order to distinguish indications of DR progress in an earlier stage. The activity of screening includes spellbinding a picture of eye fundus [5] that is examined by proficient ophthalmologists for signs of exudates or small scale micro-aneurisms [6] [7].

These days, Computer Aided Diagnostic framework (CAD) [8] [9] is accustomed so as with analyze DR by separating optic plate by assessing previously mentioned issues in the prevailing frameworks. Also, with respect to the CAD frameworks [10] to help clinicians in their procedure of basic direction has expanded, along these lines encouraging speedier and increasingly exact demonstrative choices with less discrepancy. Particularly, radiomics-driven CAD [11] [12] has turn out across the board territories of research center, in which radiomic successions including of countless picture subordinate highlights are extricated and help clinicians to plan increasingly educated choices, and offer a virtual second estimation. Then again, ordinary radiomic sequences incorporate essentially of nonexclusive, hand-crafted qualities that may be restricting in depicting typical disease practices [13] [14].

In 2016, Pratumgul et al. [15] have acquainted a method with developed programming model of CAD for screening and perceiving the thoroughness of DR in Thailand. Picture dispensation strategies with statistical topology were presented to process color pictures and distinguish five requirements foregoing be provided into ANN for recognizing. Lastly, result acquired from the examination testing set up that the presented system could offer better measurable exhibitions when contrasted and other customary plans.

In 2017, Gupta et al. [16] has introduced a methodology and framework which is based on the micro-pattern of local differences and examines structural variation in blood vessel patterns. Image-level decision of Proliferative DR (PDR) has been collected and allocated at patch level. Furthermore, the suggested strategy was examined on the wide data set of seven seventy-nine pictures from the range global clinics and public data from local clinics.

In 2018, Saleh et al. [2], proposed ensemble based classifiers with two classes of ensemble classifiers. These classifiers utilize a micro level arrangement of attributes that imply significant hazard imperatives to discover the hazard possibility of patients of creating DR. From the experimentation results, explicitness and sensitivity were found to achieve better improvement of $80 \%$. Accordingly, the proposed strategy was a first effective advancement to the production of an improved decision supportive framework which could help specialists in routine clinical practice. From above discussed literature, it is noticed that there is dire need of the learning based automated DR classification technique. The proposed system framework is discussed in next section.

\section{PROPOSED FRAMEWORK}

To overcome the limitations of existing techniques used for DR classification, we have proposed learning based DR system. Initially, the features are extracted using Tri-Concomitant conditions locally with different directions. A generalized diagram of the classification state-of-the-art approach is shown in Figure 2. Further, different directional features are concatenated to form a final feature vector. After getting the final feature vector, principle component analysis is applied to get optimal and effective features. Finally, these features are given to artificial neural network (ANN) for training with different combinations of training-testing data. Step-by-step procedure is given below.

Feature Extraction: The input DR image is processed using pixel level technique with overlapping manner. The mathematical procedure for complete feature extraction is given below.

The overlapping reference $3 \times 3$ structure is extracted from original image (I) of size $M \times N$ given as

$$
T_{\text {Input }}(p, q)=T(p+t, q+t)
$$

$$
\text { where, } t=-1: 1 ; p=2,3 \ldots \ldots . . P-1 ; q=2,3 \ldots \ldots . . Q-1
$$

The $1 \times 3$ sub structure $\left(\psi_{\theta^{0}}\right)$ extraction in given direction $\left\{\theta^{0} \in\left[0^{\circ}, 45^{\circ}, 90^{\circ}, 135^{\circ}\right]\right\}$ from each $3 \times 3$ sub block $\left(I_{3 \times 3}\right)$ is calculated as

$$
\begin{gathered}
\psi_{0^{0}}(1, i)=T_{\text {Input }}(2, i) \\
\psi_{45^{0}}(1, i)=T_{\text {Input }}(i, i) \\
\psi_{90^{0}}(1, i)=T_{\text {Input }}(i, 2) \\
\psi_{135^{5}}(1, i)=T_{\text {Input }}(4-i, i)
\end{gathered}
$$

$$
\begin{aligned}
& \text { for } \forall i=1,2,3 \\
& T\left(\psi_{\theta^{0}}\right)=\left\{\begin{array}{l}
1 \\
2 \\
3 \\
4 \\
5 \\
6 \\
7
\end{array}\right. \\
& \left\langle\psi_{\theta^{0}}(1,3)>\psi_{\theta^{0}}(1,2)\right\rangle \&\left\langle\psi_{\theta^{0}}(1,2)>\psi_{\theta^{0}}(1,1)\right\rangle \\
& \left\langle\psi_{\theta^{0}}(1,3)<\psi_{\theta^{0}}(1,2)\right\rangle \&\left\langle\psi_{\theta^{0}}(1,2)<\psi_{\theta^{0}}(1,1)\right\rangle \\
& \left\langle\psi_{\theta^{0}}(1,3)>\psi_{\theta^{0}}(1,1)\right\rangle \&\left\langle\psi_{\theta^{0}}(1,2)<\psi_{\theta^{0}}(1,3)\right\rangle \\
& \left\langle\psi_{\theta^{0}}(1,3)<\psi_{\theta^{0}}(1,1)\right\rangle \&\left\langle\psi_{\theta^{0}}(1,2)<\psi_{\theta^{0}}(1,1)\right\rangle \\
& \left\langle\psi_{\theta^{0}}(1,3)>\psi_{\theta^{0}}(1,1)\right\rangle \&\left\langle\psi_{\theta^{0}}(1,2)>\psi_{\theta^{0}}(1,3)\right\rangle \\
& \left\langle\psi_{\theta^{0}}(1,3)<\psi_{\theta^{0}}(1,1)\right\rangle \&\left\langle\psi_{\theta^{0}}(1,2)>\psi_{\theta^{0}}(1,3)\right\rangle \\
& \text { else } \\
& T_{\theta_{p, q}^{0}}^{1}\left(g_{i}\right)=T_{\theta_{p, q}^{0}}^{1}\left(g_{i}\right)-T_{\theta_{p, q}^{0}}^{1}\left(g_{c}\right) ; \\
& T_{\theta_{p, q+1}^{0}}^{1}\left(g_{i}\right)=T_{\theta_{p, q+1}^{0}}^{1}\left(g_{i}\right)-T_{\theta_{p, q}^{0}}^{1}\left(g_{c}\right) ;
\end{aligned}
$$


Where, $\theta^{0} \in\left[0^{0}, 45^{0}, 90^{0}, 135^{\circ}\right], i=1,2, \ldots \ldots . . p$

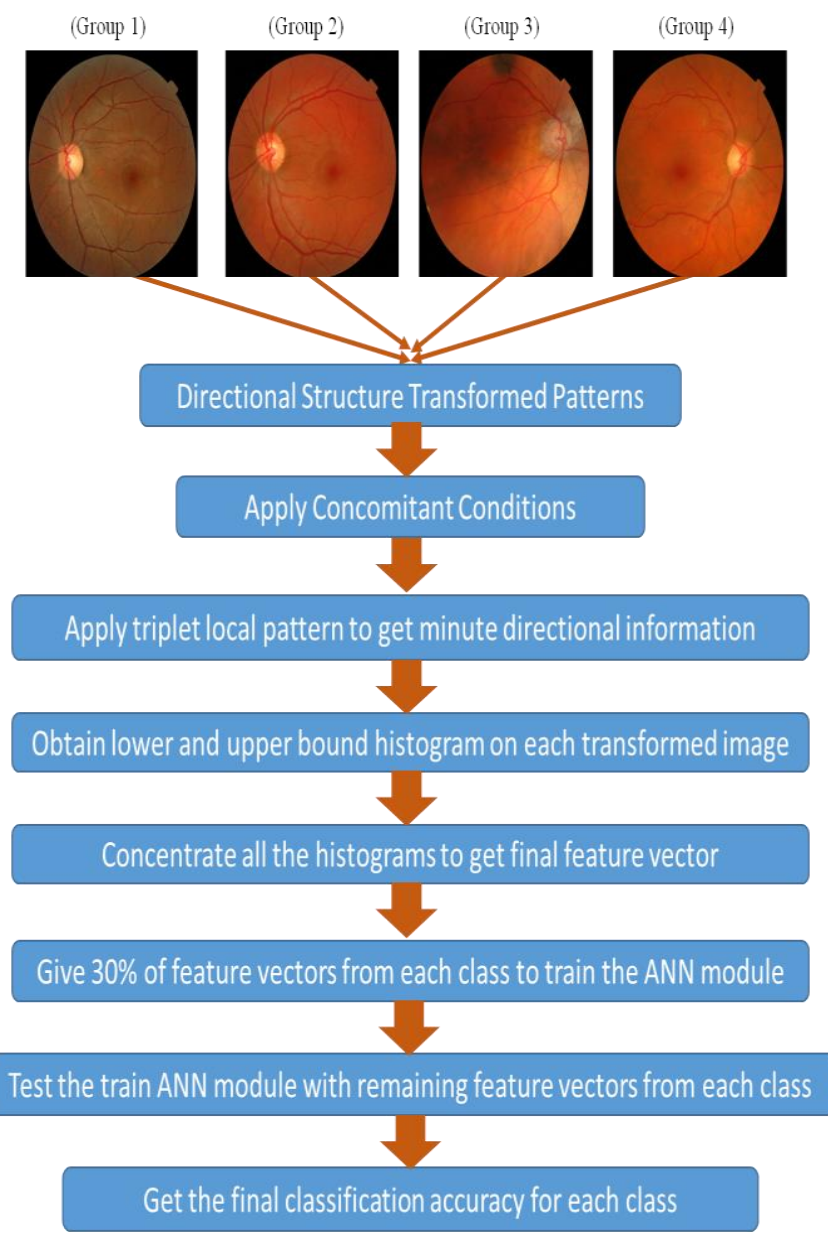

Fig. 3. Block diagram of the proposed classification approach for diabetic retinopathy

After first order derivative calculation, we code them based on the sign of derivatives as follows

$$
\begin{gathered}
T_{\theta_{p, q}^{0}}^{1}\left(g_{i}\right)=f_{1}\left(T_{\theta_{p, q}^{0}}^{1}\left(g_{i}\right)\right) \\
T_{\theta_{p, q+1}^{0}}\left(g_{i}\right)=f_{1}\left(T_{\theta_{p, q+1}^{0}}\left(g_{i}\right)\right)
\end{gathered}
$$

Where, $\theta^{0} \in\left[0^{0}, 45^{0}, 90^{\circ}, 135^{\circ}\right]$

Above mathematical procedure gives the three-valued image, which further used to find the concomitant value between the elements as

$$
\operatorname{Feat}(m, n)=\left[\begin{array}{c}
f_{2}\left(T_{\theta_{p, q}^{0}}^{1}\left(g_{1}\right), T_{\theta_{p, q+1}^{0}}^{1}\left(g_{1}\right)\right), \\
f_{2}\left(T_{\theta_{p, q}^{0}}^{1}\left(g_{2}\right), T_{\theta_{p, q+1}^{0}}^{1}\left(g_{2}\right)\right), \ldots \\
\ldots . f_{2}\left(T_{\theta_{p, q}^{0}}^{1}\left(g_{p}\right), T_{\theta_{p, q+1}^{0}}^{1}\left(g_{p}\right)\right)
\end{array}\right]
$$

Where, $\theta^{0} \in\left[0^{0}, 45^{0}, 90^{\circ}, 135^{\circ}\right]$,

$$
\begin{array}{r}
m=3,4, \ldots . . . M-2, n=3,4, \ldots \ldots . . N-2 \\
f_{2}(x, y)=\left\{\begin{array}{ccc}
1 & \text { if } & x=y=1 \\
-1 & \text { if } & x=y=-1 \\
0 & \text { else }
\end{array}\right. \\
\text { FeatVector }_{\theta_{L T P}^{0}}=\sum_{i=1}^{8} \text { Feat }_{\theta_{L T P}^{0}} \times\left(2^{i}\right) \\
\text { FeatVector }_{\theta_{U T P}^{0}}=\sum_{i=1}^{8} \text { Feat }_{\theta_{U T P}^{0}} \times\left(2^{i}\right)
\end{array}
$$

Where, $\theta^{0} \in\left[0^{0}, 45^{0}, 90^{\circ}, 135^{\circ}\right]$
After calculating entire feature for whole image, histogram of image is estimated to form a final feature vector as

$$
\begin{aligned}
& \text { Hist }_{\text {Featrect } \theta_{\text {ITP }}}^{\theta^{0}}(p)=\sum_{m=1}^{M} \sum_{n=1}^{N} f_{2}\left(\text { FeatVect }_{\theta_{\text {LIP }}^{0}}(m, n), p\right) \text {; } \\
& \text { Hist }_{\text {FearlecturP }}^{\theta^{0}}(p)=\sum_{m=1}^{M} \sum_{n=1}^{N} f_{2}\left(\text { FeatVect }_{\theta_{\text {UTP }}^{0}}(m, n), p\right) \\
& f_{2}(x, y)= \begin{cases}1 & x=y \\
0 & \text { else }\end{cases}
\end{aligned}
$$

Upper and Lower histogram of proposed feature in all direction are concatenate to form final feature vector as.

$$
\text { Hist }_{\text {FeatVect }}=\left[\text { Hist }_{\text {FeatVect }}^{\theta_{\text {UTP }}} \quad \text { Hist }_{\text {FeatVect }_{\text {LTP }}^{\theta^{0}}}^{\theta^{0}}\right]
$$

Thus, using above mentioned mathematical procedure the final feature vector is formed. Further, these feature vectors are processed through PCA to get effective and efficient feature for learning. The block diagram of the proposed system framework is given in Figure 3.

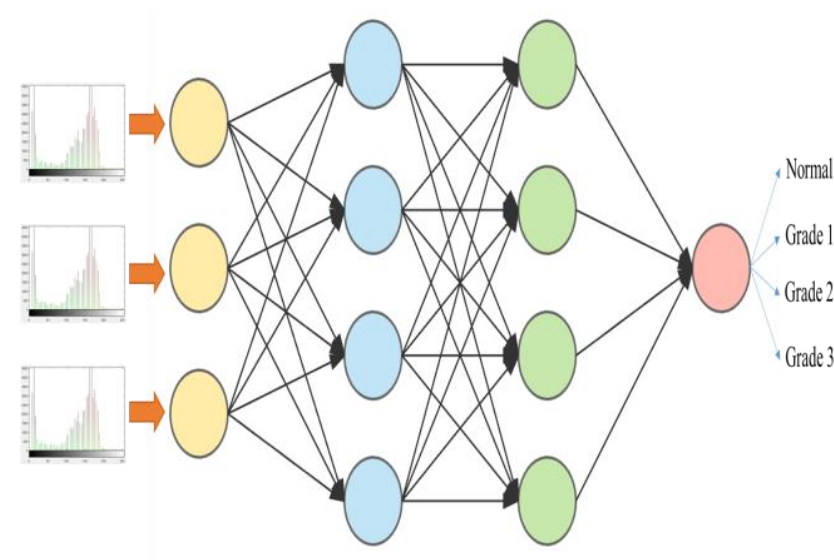

Extracted Features input layer hidden layer 1 hidden layer 2 output layer

Fig. 4. Generlasid ANN network for DR classification

Principle Component Analysis: Principal component analysis (PCA) is a statistical method which uses an orthogonal transformation to translate a set of interpretation of probably correlated variable into a set of values of linearly uncorrelated variables called principal components. This change is particular so that the distinct segment has the greatest likely fluctuation and each resulting segment thus has the most extreme difference conceivable under the imperative that it is symmetrical to the first segments. The resultant vectors are an uncorrelated symmetrical premise set. PCA is receptive to the overall scaling of the one of a kind factors. PCA basically utilized for the dimension reduction of feature vector which are got from the feature extraction. It will allow the reduction of computational complexity in the classification process.

Artificial Neural Network: As learning based techniques gaining more demand in day-to-day life for different computer vision applications, learning based classification approach is used in proposed system. The main advantage for learning based system is able to produce significant results in various computer vision application like image de-hazing, image retrieval, moving object segmentation, depth estimation, human action recognition, automated video applications like video surveillance, traffic monitoring, etc. 
A generalized network for ANN is given in Figure 3. We have used ANN inbuilt command in MATLAB-2017b. Proposed ANN framework is designed for DR classification in to four type's i.e. Normal, Grade I, Grade II and Grade III. As extracted features are very effective and efficient, the proposed ANN network is able to get best classification results.

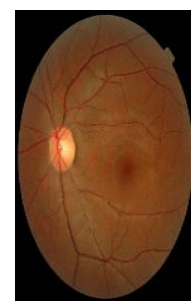

(Group 1)

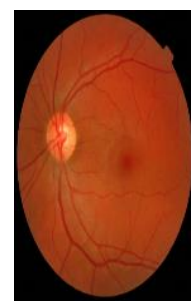

(Group 2)

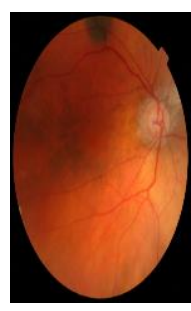

(Group 3)

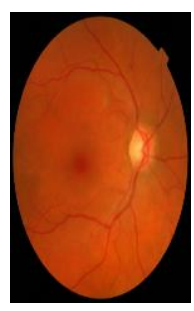

(Group 4)

Fig. 5. Sample images from MESSIDOR DR image

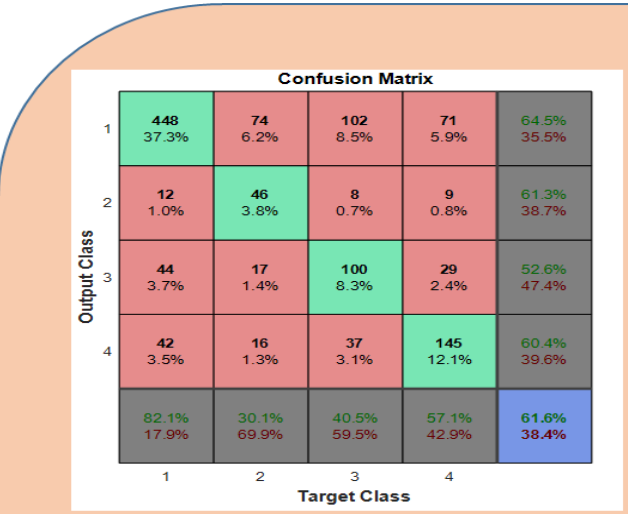

(a) $\mathbf{5 0} \%-\mathbf{4 0} \%$ Training Testing

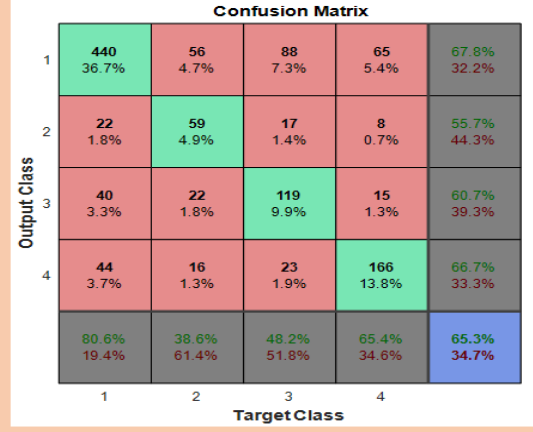

(c) $70 \%-20 \%$ Training Testing database

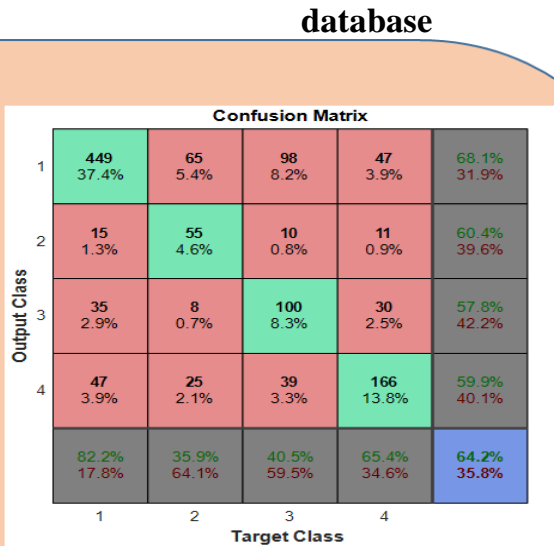

(b) $60 \%-30 \%$ Training Testing

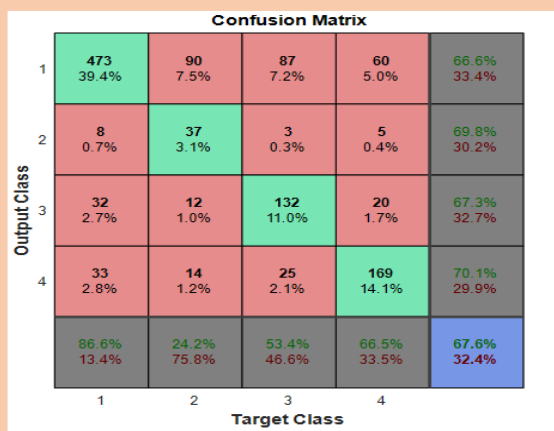

(d) $80 \%-10 \%$ Training Testing

Fig. 6. Accuracy comaprision with combinations of different training-testing data

\section{RESULTS AND DISCUSSION}

To examine the effectiveness of the proposed approach MESSIDOR database is used. This database comprises of 1200 DR images in total with four different groups. The sample images from each category are given in Figure 5. The average precision is used as evaluation parameter. The results obtained by proposed method are illustrated in Figure 6. The training data variation approach is used in proposed approach. Means, the training testing data is varied and accuracy is examined. Thus, from results, it is observed that the proposed method able to give the good classification accuracy for DR images. Further, this proposed approach may be used for natural image classification as well as other modalities of medical images like MRI, Brain, Breast cancer, etc.

\section{CONCLUSION}

In this paper, Transformed Directional Tri Concomitant Triplet Patterns with Artificial Neural Network is proposed for Diabetic Retinopathy Classification. The proposed pattern make use of local directional information with concomitant conditions to extract the effective and meaningful features. Further these features are used train the ANN network for classification. The classification accuracy is measured in terms of average precision with different percentages of training and testing data. Proposed method is achieved improved performance with $80 \%$ of data training and remaining data for testing.

\section{ACKNOWLEDGMENT}

Authors would like to thanks to Pimpri Chinchwad Education Trust's Pimpri Chichwad College of Engineering \& Research, Ravet, Pune for their encouragement and valuable support during this research work.

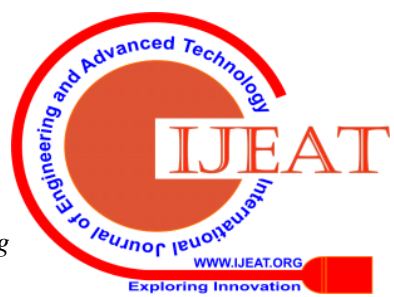




\section{REFERENCES}

1. Roberto Rosas-Romero, Jorge Martínez-Carballido, Jonathan Hernández-Capistrán, Laura J. Uribe-Valencia, “A method to assist in the diagnosis of early diabetic retinopathy: Image processing applied to detection of microaneurysms in fundus images", Computerized Medical Imaging and Graphics, vol. 44, pp. 41-53, September 2015.

2. Emran Saleh, Jerzy Błaszczyński, Antonio Moreno, Aida Valls, Roman Słowiński, "Learning ensemble classifiers for diabetic retinopathy assessment", Artificial Intelligence in Medicine, vol. 85, pp. 50-63, April 2018.

3. Manuel E. Gegundez-Arias, Diego Marin, Beatriz Ponte, Fatima Alvarez, Jose M. Bravo, "A tool for automated diabetic retinopathy pre-screening based on retinal image computer analysis", Computers in Biology and Medicine, vol. 88, pp. 100-109, 1 September 2017.

4. M. Nalini., B.V. Raghavulu, A. Annapurna, P. Avinash, Wasim, "Correlation of various serum biomarkers with the severity of diabetic retinopathy", Diabetes \& Metabolic Syndrome: Clinical Research \& Reviews, vol. 11, Supplement 1, pp. 451-454, November 2017.

5. M.D. Pinazo-Durán, K. Shoaie-Nia, S.M. Sanz-González, J. Raga-Cervera, "Identification of new candidate genes for retinopathy in type 2 diabetics. Valencia Study on Diabetic Retinopathy (VSDR). Report number 3", 23 March 2018.

6. Peter L. Nesper, Brian T. Soetikno, Hao F. Zhang, Amani A. Fawzi, "OCT angiography and visible-light OCT in diabetic retinopathy", Vision Research, vol. 139, pp. 191-203, October 2017.

7. Bruna Letícia da Silva Pereira, Evelise Regina Polina, Daisy Crispim, Renan Cesar Sbruzzi, Kátia Gonçalves dos Santos, "Interleukin-10 $-1082 \mathrm{~A}>\mathrm{G}$ (rs1800896) polymorphism is associated with diabetic retinopathy in type 2 diabetes", Diabetes Research and Clinical Practice, vol. 138, pp. 187-192, April 2018.

8. Muhammad Adam, Eddie Y.K. Ng, Jen Hong Tan, Marabelle L. Heng, U. Rajendra Acharya, "Computer aided diagnosis of diabetic foot using infrared thermography: A review", Computers in Biology and Medicine, vol. 91, pp. 326-336, 1 December 2017.

9. Florin Gorunescu, Smaranda Belciug, "Boosting backpropagation algorithm by stimulus-sampling: Application in computer-aided medical diagnosis", Journal of Biomedical Informatics, vol. 63, pp. 74-81, October 2016

10.Melih Kandemir, Fred A. Hamprecht, "Computer-aided diagnosis from weak supervision: A benchmarking study", Computerized Medical Imaging and Graphics, vol. 42, pp. 44-50, June 2015.

11.Jay Desai, Gretchen Taylor, Gabriela Vazquez-Benitez, Sara Vine, Patrick J. O'Connor, "Financial incentives for diabetes prevention in a Medicaid population: Study design and baseline characteristics", Contemporary Clinical Trials, vol. 53, pp. 1-10, February 2017.

12.Lilla Bonanno, Silvia Marino, Placido Bramanti, Fabrizio Sottile, "Validation of a Computer-Aided Diagnosis System for the Automatic Identification of Carotid Atherosclerosis", Ultrasound in Medicine \& Biology, vol. 41, no. 2, pp. 509-516, February 2015.

13.U. Rajendra Acharya, K. Sudarshan Vidya, Dhanjoo N. Ghista, Wei Jie Eugene Lim, Meena Sankaranarayanan, "Computer-aided diagnosis of diabetic subjects by heart rate variability signals using discrete wavelet transform method", Knowledge-Based Systems, vol. 81, pp. 56-64, June 2015.

14.Hiroshi Fujita, Yoshikazu Uchiyama, Toshiaki Nakagawa, Daisuke Fukuoka, Xiangrong Zhou, "Computer-aided diagnosis: The emerging of three CAD systems induced by Japanese health care needs", Computer Methods and Programs in Biomedicine, vol. 92, no. 3, pp. 238-248, December 2008.

15.Weeagul Pratumgul, Worawat Sa-ngiamvibool, "The Prototype of Computer-Assisted for Screening and Identifying Severity of Diabetic Retinopathy Automatically from Color Fundus Images for mHealth System in Thailand", Procedia Computer Science, vol. 86, pp. 457-460, 2016

16. Garima Gupta, S. Kulasekaran, Keerthi Ram, Niranjan Joshi, Rashmin Gandhi, "Local characterization of neovascularization and identification of proliferative diabetic retinopathy in retinal fundus images", Computerized Medical Imaging and Graphics, vol. 55, pp. 124-132, January 2017.

\section{AUTHORS PROFILE}

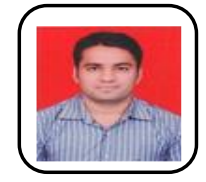

Santosh N. Randive received the B.E. (Electronics \& Telecommunication) degree from Savitribai Phule Pune University (Formerly University of Pune ), Pune, Maharashtra, India, in 2010, M.Tech. degree from SGGS Institute of Engineering and Technology, Nanded affiliated to Swami Ramanand Teerth Marathwada
University Nanded, India in 2012.He is presently pursuing his Ph.D. from Department of Electronics \& Communication Engineering, Koneru Lakshmaiah Education Foundation, Green Fields, Vaddeswaram, Guntur, Andhra Pradesh India. His research interests include Medical Image Processing and Computer Vision.

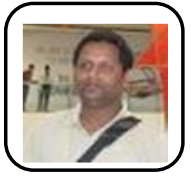

Ranjan K. Senapati received his M.Tech degree from NIT Warangal, and Ph.D. degree from NIT Rourkela, India in 2004 and 2014 respectively. At present, he is working as a professor in the department of electronics and communication engineering, Koneru Lakshmaiah Education Foundation, Green Fields, Vaddeswaram, Guntur, Andhra Pradesh India. He has published over 30 papers in refereed journals and conference proceedings. He is currently guiding 8 Ph.D. scholars. His research interests include distributed arithmetic architecture implementation of transform algorithms, development of image and video compression algorithms for JPEG 2000, H.264, and HEVC standards. He is a member various professional societies such as ISTE, IETE and IEEE. 